\title{
Structural and Kinetic Comparison of Recombinant Human Single- and Two-Chain Tissue Plasminogen Activator
}

\author{
Joseph Loscalzo \\ Vascular and Cardiology Divisions, Brigham and Women's Hospital and Harvard Medical School, Boston, Massachusetts 02115
}

\begin{abstract}
We examined the similarities and differences in conformation between recombinant human single-chain tissue plasminogen activator (sct-PA) and two-chain tissue plasminogen activator (tct-PA), and compared these structural data with measurements of enzymatic activity. The intrinsic protein fluorescence of native tct-PA was $54 \%$ that of sct-PA. Differences in steady state protein fluorescence were also noted with denaturation of these plasminogen activators, as well as in the quenching of intrinsic fluorescence of the reduced, alkylated species by iodide. Using the chromogenic substrate H-D-isoleucyl-L-prolyl-L-arginine-p-nitroanilide (S-2288), the catalytic efficiency of sct-PA was found to be $26 \%$ that of tet-PA, and this was primarily a reflection of the difference in $K_{m}$. On addition of soluble fibrin monomer prepared with the tetrapeptide glycylL-prolyl-L-arginyl-L-proline (GPRP), the catalytic efficiency of both species increased by 13-fold for sct-PA and by 3.5-fold for tct-PA to approximately the same value. Using the fluorophore eosin iodoacetamide covalently coupled to the single free cysteine in the molecule, Cys 83 , the microenvironment of the fibrin-binding site located near this residue was studied. On addition of soluble fibrin monomer to eosin-labeled tct-PA, no effect on eosin fluorescence was noted. Eosin-labeled tct-PA had 16\% less eosin fluorescence than did sct-PA and on addition of soluble fibrin monomer to eosin-labeled sct-PA, a decrease in eosin fluorescence, approaching that of eosin coupled to tct-PA, was observed. Together, these structural and kinetic data suggest that sct-PA undergoes a conformational change on binding to fibrin monomer that leads to dramatic differences in catalytic efficiency of the single-chain species. In so doing, sct-PA bound to fibrin assumes the kinetic profile of tct-PA bound to fibrin.
\end{abstract}

\section{Introduction}

Human tissue plasminogen activator (t-PA) ${ }^{1}$ is an essential enzymatic component in the constellation of proteins that

Address reprint requests to Dr. Joseph Loscalzo, Division of Vascular Medicine and Atherosclerosis, Department of Medicine, Brigham and Women's Hospital, 75 Francis Street, Boston, MA 02115.

Received for publication 4 January 1988 and in revised form 10 May 1988.

1. Abbreviations used in this paper: eos-t-PA, 5-(iodoacetamido)eosinlabeled tissue plasminogen activator; FM-Matrex, Matrex bead-immobilized fibrin monomer; GPRP, glycyl-L-prolyl-L-arginyl-L-proline; PBST, phosphate-buffered saline with $0.006 \%$ Tween 80 ; PLL, poly-L-

J. Clin. Invest.

(c) The American Society for Clinical Investigation, Inc. 0021-9738/88/10/1391/07 \$2.00

Volume 82, October 1988, 1391-1397 promote fibrinolysis (1). This enzyme is synthesized by endothelial cells in a single-chain form (sct-PA) and, by analogy with other serine proteases (2), can be converted to a two-chain form (tct-PA) by proteolysis at Arg 275. Controversy exists in the literature about the differences in activity and fibrin specificity between sct-PA and tct-PA. Three groups have reported that tct-PA is considerably more active than sct-PA (3-5), and these investigators have suggested that any measured activity in preparations of sct-PA represent contamination by small amounts of the two-chain form. Other investigators have reported that sct-PA does have significant intrinsic enzymatic activity (6-11). Interpretation of these data is complicated by several factors, including the method used to measure t-PA activity, the source of t-PA, and the method used to measure activation by fibrin. Rijken and colleagues (8) sought to overcome the problem of conversion by plasmin of sct-PA to tctPA using high concentrations of aprotinin. They measured the plasmin produced by t-PA using quantitative autoradiography but were unable to measure directly the $K_{\mathrm{m}}$ and $V_{\max }$ in the absence of fibrin because they could not achieve substrate saturation with the native substrate. Tate and colleagues (5) overcame this latter problem using a chromogenic substrate and synthesized a noncleavable sct-PA using site-directed mutagenesis, but failed to measure activation in the presence of fibrin.

In the experiments presented here, we compare the activity of native sct-PA with that of tct-PA in the presence and absence of fibrin. We compare the activity of sct-PA with that of tct-PA against the relatively specific chromogenic substrate H-D-isoleucyl-L-prolyl-L-arginine-p-nitroanilide (S-2288), thereby avoiding the complications engendered by evolving plasmin activity in the assay medium. We use soluble fibrin monomer prepared according to the method of Laudano and Doolittle (12) with modification (13) to assess directly the effects of fibrin on t-PA activity. As a central aspect of this paper, we correlate these kinetic studies with conformational studies to assess the relationship between structure and function of these related molecules. Our data indicate that native sct-PA and tct-PA have different conformations and different enzymatic properties in the absence of fibrin, but that fibrin binding induces conformational changes in sct-PA that are accompanied by changes in enzymatic activity essentially similar to that of tct-PA.

\section{Methods}

Materials. sct-PA was obtained from Genentech, Inc., South San Francisco, CA. S-2288, H-D-valyl-L-leucine-L-lysine-p-nitroanilide

lysine; PN-Sepharose, plasmin immobilized on Sepharose 4B; PPACK, D-phenyalanyl-L-prolyl-L-arginine chloromethylketone. $2 \mathrm{HCl}$; sct-PA, single-chain tissue plasminogen activator; SFM, soluble fibrin monomer; S-2288, H-D-isoleucyl-L-prolyl-L-arginine-p-nitroanilide; S-2251, H-D-valyl-L-leucyl-L-lysine-p-nitroanilide; tct-PA, twochain tissue plasminogen actirator. 
(S-2251), human plasminogen, human plasmin, and human fibrinogen were purchased from KABI Vitrum, Stockholm, Sweden. Matrex Pel 102 beads were obtained from Amicon Corp., Danvers, MA. Plasminogen-free bovine thrombin was purchased from Miles Pharmaceuticals, Naperville, IL. Aprotinin and glycyl-L-prolyl-L-arginyl-Lproline (GPRP) were obtained from Sigma Chemical Co., St. Louis, MO. D-Phenylalanyl-L-prolyl-L-arginine chloromethylketone. $2 \mathrm{HCl}$ (PPACK) was purchased from Calbiochem-Behring Corp., La Jolla, CA. Iodo-beads were obtained from Pierce Chemical Corp., Rockford, IL. 5-(Iodoacetamido)eosin was purchased from Molecular Probes, Inc., Junction City, OR. $\mathrm{Na}\left[{ }^{125} \mathrm{I}\right]$ was obtained from Amersham Corp., Arlington Heights, IL. $\left[{ }^{14} \mathrm{C}\right]$ lodoacetamide was obtained from New England Nuclear, Boston, Ma. Cyanogen bromide-activated Sepharose 4B was purchased from Pharmacia Fine Chemicals, Uppsala, Sweden. All other chemicals were reagent grade or better. Deionized water was used throughout.

Preparation of plasmin-Sepharose. Purified plasmin was coupled to cyanogen bromide-activated Sepharose 4B in $0.1 \mathrm{M} \mathrm{NaHCO}_{3}, \mathrm{pH} 8.3$, $0.5 \mathrm{M} \mathrm{NaCl} .900 \mu \mathrm{g}$ plasmin was added to $5 \mathrm{ml}$ of activated beads. The reaction was allowed to proceed at room temperature for $2 \mathrm{~h}$, after

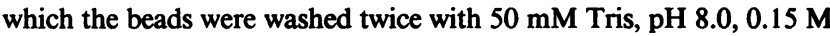
$\mathrm{NaCl}$, then alternately with $0.1 \mathrm{M}$ sodium acetate, $\mathrm{pH} 4.0,0.5 \mathrm{M} \mathrm{NaCl}$, and $0.1 \mathrm{M} \mathrm{NaHCO}_{3}, \mathrm{pH} 8.3,0.5 \mathrm{M} \mathrm{NaCl}$. The washed plasmin-coupled beads were finally suspended in $10 \mathrm{mM}$ sodium phosphate, $\mathrm{pH}$ $7.4,0.15 \mathrm{M} \mathrm{NaCl}, 0.006 \%$ Tween 80 (PBST) and stored at $4^{\circ}$. When prepared in this fashion, $25 \mu \mathrm{l}$ of a $30 \%$ slurry of plasmin immobilized on Sepharose 4B (PN-Sepharose) routinely hydrolyzed $1.0 \mathrm{mM} \mathrm{S}-2251$ at $37^{\circ} \mathrm{C}$ at a rate of $0.077 \mathrm{ODU} / \mathrm{min}$.

Conversion of sc-tPA to tct-PA. Predominantly sct-PA (76\% by $\mathrm{NH}_{2}$-terminal amino acid analysis using a gas phase sequenator [Applied Biosystems, Foster City, CA] at the Peptide Facility of Brigham and Women's Hospital, as well as by electrophoretic analysis and densitometry) was converted to tct-PA by incubating $50 \mu \mathrm{l}$ of the PNSepharose slurry with $1 \mathrm{ml}$ of $0.9 \mathrm{mg} / \mathrm{ml}$ sct-PA at room temperature for $2 \mathrm{~h}$ with rocking. At the end of the incubation, the PN-Sepharose was removed by centrifugation at $8,700 \mathrm{~g}$ for $1 \mathrm{~min}$ in an Eppendorf microfuge.

Completeness of conversion was monitored by SDS-PAGE performed in the presence of 2-mercaptoethanol (2-ME). Under the conditions of our assay, conversion was complete at $2 \mathrm{~h}$.

Radioiodination of $t-P A$. sct-PA was radioiodinated using Iodobeads. One Iodo-bead was incubated with $0.5 \mathrm{mCi}$ of $\mathrm{Na}\left[{ }^{125} \mathrm{I}\right]$ at $25^{\circ} \mathrm{C}$ for $15 \mathrm{~min}$. To this was added $1.0 \mathrm{ml}$ of $0.1 \mathrm{mg} / \mathrm{ml} \mathrm{sct-PA}$ in PBST and the incubation continued for $20 \mathrm{~min}$ with gentle rocking. The protein solution was then applied to a $10 \times 0.7 \mathrm{~cm}$ Sephadex G-25 column that had been washed with a solution of $5 \mathrm{mg} / \mathrm{ml}$ BSA. $120.3-\mathrm{ml}$ fractions were collected and assayed for total and 25\% TCA-precipitable radioactivity. Routinely, column fractions four through six contained maximal protein-bound counts with a specific activity of $\sim 0.1-0.2$ $\mu \mathrm{Ci} / \mu \mathrm{g}$. These fractions were either used immediately or stored at $4^{\circ} \mathrm{C}$ for up to $1 \mathrm{wk}$ without appreciable loss of protein-bound counts. In our hands, radioiodination did not affect either the enzymatic activity of t-PA or the enhancement of that activity by fibrin. When radiolabeled tct-PA was required for an experiment, ${ }^{125} \mathrm{I}$-sct-PA was treated with PN-Sepharose as described above.

Labeling of $t-P A$ with 5-(iodoacetamido)-eosin. Eos-t-PA was produced by incubating sct-PA with a 25-fold molar excess of fluorophore (solubilized in acetone) in PBST at room temperature in the dark for 2 h. Excess unreacted fluorophore was removed by extensive dialysis at $4^{\circ} \mathrm{C}$ in the dark. t-PA has only a single cysteine (Cys 83 ) that is not involved in a disulfide bond (14), and under the conditions of labeling (pH > 7.0), only this free thiol will react with the iodoacetamide moiety. The extent of incorporation of label was assessed by the method of Franzen and colleagues (15). In addition, the labeled molecule was treated with $\left[{ }^{14} \mathrm{C}\right]$ iodoacetamide in an effort to measure directly unreacted cysteine groups. Compared with the native sct-PA molecule, in which $0.97 \mathrm{~mol}$ of $\left[{ }^{14} \mathrm{C}\right]$ iodoacetamide were bound per mol of protein, $0.04 \mathrm{~mol}$ of $\left[{ }^{14} \mathrm{C}\right]$ iodoacetamide were bound per mol of
eos-sct-PA, confirming the essential completeness of the labeling reaction. Labeling t-PA with eosin iodoacetamide had no effect on intrinsic enzymatic activity against S-2288 (see below) or on binding to fibrin (see below).

Preparation of Matrex bead-immobilized fibrin monomer (FMMatrex). FM-Matrex was prepared essentially according to the method of Loscalzo and colleagues (13). Fibrinogen was covalently coupled to solid beads of polymerized acrylonitrile with free carboxyl and $\mathrm{N}$-hydroxysucciminide carboxylate ester groups on the surface (Matrex 102) essentially according to the method of Coller (16). Fibrinogen covalently coupled to the beads was then converted to fibrin monomer by incubation with $4 \mathrm{U} / \mathrm{ml}$ of bovine thrombin for $1 \mathrm{~h}$ at $25^{\circ} \mathrm{C}$. At the end of the incubation, PPACK was added to a concentration of $48 \mu \mathrm{M}$ to inhibit further thrombin activity. The beads were then washed three times with PBST and stored for up to 2 wk at $4^{\circ} \mathrm{C}$ without a detectable loss of bound protein. Using ${ }^{125} \mathrm{I}$-fibrinogen, $\sim 4.0 \times 10^{5}$ molecules of fibrinogen bound per 3- $\mu \mathrm{m}$-diam bead with a $95 \%$ coupling efficiency. Because of steric constraints induced by the coupling process, fibrin monomer was unable to assemble into polymer on the bead surface, and this immobilized fibrin monomer bound to Matrex was used in the binding experiments described below.

Preparation of soluble fibrin monomer (SFM). SFM was prepared as described previously $(12,13)$, starting with human fibrinogen purified free of plasminogen by standard methods $(17,18)$. The SFM was maintained in the monomeric state for up to $1.5 \mathrm{~h}$ at room temperature in the presence of $2 \mathrm{mM}$ GPRP. In the absence of GPRP, greater than $95 \%$ of the fibrinogen was clottable.

Measurement of $t-P A$ activity. The enzymatic activity of t-PA was assessed using the relatively specific chromogenic substrate S-2288. Substrate hydrolysis was measured spectrophotometrically with a Gilford Response UV/Vis Spectrophotometer (CIBA-Corning, Oberlin, $\mathrm{OH})$. Activity was measured at $37^{\circ} \mathrm{C}$ in PBST using substrate concentrations ranging from 0.075 to $1.5 \mathrm{mM}$ and a t-PA concentration of $9.20 \times 10^{-8} \mathrm{M}$. Kinetic parameters were determined from the initial rates by double-reciprocal plot analysis. Since the sct-PA preparation contained $24 \%$ tct-PA by amino-terminal analysis, constants for sctPA were determined by correcting the reaction velocity at each substrate concentration for the percentage contamination with tct-PA.

Ultraviolet (UV) spectrophotometry. Absorption measurements were performed in a Gilford Response UV/Vis spectrophotometer. Spectra were recorded at $25^{\circ} \mathrm{C}$ in PBST. Difference spectra were obtained by subtracting spectra taken at $\mathrm{pH} 7.4$ from those recorded at pH 11.5 .

Fluorescence spectrophotometry. Fluorescence measurements were performed on a spectrofluorometer (Fluorolog 2 model F111X; Spex Industries, Inc., Edison, NJ) equipped with a thermostatted cell holder. The absorbance of solutions was $<0.1$ at exciting wavelengths. A reference cell containing rhodamine $B$ was used to correct for variation and nonlinearity in the excitation source.

Quantum yields were derived from fluorescence intensity measurements at 280 or $295 \mathrm{~nm}$, using the method of Parker and Rees (19):

$A_{\mathrm{p}} / A_{\mathrm{r}}=Q_{\mathrm{p}} D_{\mathrm{p}} /\left(Q_{\mathrm{r}} D_{\mathrm{r}}\right)$

where $A$ is the area under the fluorescence spectrum and $D$ is the optical density at the exciting wavelength. The subscripts $p$ and $r$ refer to protein and reference solution, respectively. A value of 0.20 for $Q_{\text {trp }}$ was used $(20,21)$.

Since tyrosine quantum yields cannot be measured directly from recorded spectra, they were calculated from the protein and tryptophan quantum yields, according to the method of Kronman and Holmes (21):

$Q_{\mathrm{tyr}}=Q_{\mathrm{tyr}}^{\prime} /\left[l+(m / n)\left(\epsilon_{\mathrm{trp}} / \epsilon_{\mathrm{tyr}}\right)\right]$

where $m$ and $n$ are the number of tryptophan and tyrosine residues per protein molecule, respectively, $\epsilon_{\text {trp }} / \epsilon_{\mathrm{tyr}}$ is the ratio of the extinction coefficients of solutions of the free amino acids, $Q_{\mathrm{tyr}}^{\prime}$ is estimated from

$Q_{\mathrm{tyr}}^{\prime}=Q_{\mathrm{pro}}-Q_{\mathrm{trp}}^{\prime}$ 
and $Q_{\mathrm{trp}}^{\prime}$ is derived from the amplitude $(F)$ and area $(A)$ of the emission spectra as

$Q_{\mathrm{trp}}=Q_{\mathrm{trp}}^{\prime}\left[(F / A)_{\lambda=280} /(F / A)_{\lambda=295}\right]$

Binding of $t-P A$ to FM-Matrex. The binding of sct-PA and tct-PA to FM-Matrex was measured at $25^{\circ} \mathrm{C}$ in PBST. Increasing concentrations of radioiodinated t-PA were incubated with $1 \times 10^{4} \mathrm{FM}$-Matrex beads $/ \mu l$ in a 300- $\mu$ l assay volume of PBST at room temperature with rocking for $20 \mathrm{~min}$. At the end of the incubation period, the beads were collected by centrifugation at $8,700 \mathrm{~g}$ for $2 \mathrm{~min}$, washed twice with 1.5 $\mathrm{ml}$ of PBST, after which bound radioactivity was measured in a RIAGamma Counter Model 1274 (LKB Instruments, Gaithersburg, MD). Nonspecifically bound t-PA was distinguished from specifically bound t-PA by addition of a 20-fold excess of unlabeled t-PA.

Reduction and alkylation of $t-P A$. Both sct-PA and tct-PA were reduced and alkylated by carboxyamidation in the following manner. The proteins were incubated at room temperature for $1 \mathrm{~h}$ with $50 \mathrm{mM}$ 2-ME in PBST without any denaturing agents, after which $60 \mathrm{mM}$ iodoacetamide was added in the same buffer. Extensive dialysis against PBST was then performed to remove unbound reactants. Under these conditions, essentially all of the $\mathbf{3 5}$ sulfhydryl groups per molecule of t-PA were exposed (only one of which is not disulfide linked in the native molecule) as assayed by the 5,5'-dithiobis-(2-nitrobenzoic acid) method $(22,23)$. A molar extinction coefficient of $14,200 \mathrm{M}^{-1} / \mathrm{cm}^{-1}$ for the nitrothiophenolate ion was used to calculate the concentration of free sulfhydryl groups (24). Precipitating t-PA and showing that 5,5'-dithiobis-(2-nitrobenzoic acid) failed to react with the modified protein demonstrated that alkylation was complete under these conditions.

PAGE. SDS-PAGE was performed as described by Weber and Osborne (25) and modified by Laemmli (26) using $7.5 \%$ gels. Gels were stained with Coomassie brilliant blue in 50\% methanol and 5\% acetic acid, and destained by diffision. Molecular weight standards were processed similarly and apparent molecular weights $\left(M_{\mathrm{r}}\right)$ were estimated by interpolation. Quantitative reflectance densitometry was performed using a videodensitometry system (model 620; Bio-Rad Laboratories, Richmond, CA)

Protein determinations. Protein concentrations were determined according to the method of Lowry and colleagues (27).

\section{Results}

\section{Secondary and tertiary structure}

$U V$ absorption. The absorbance difference spectra in the farUV region for sct-PA and tct-PA are shown in Fig. 1. These spectra are similar, showing positive bands at 244 and $296 \mathrm{~nm}$. The positive band at the longer wavelength is probably due to vibrational structure (28).

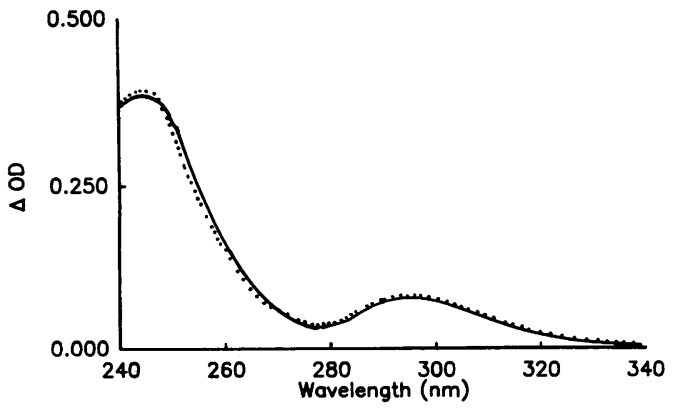

Figure 1. Difference absorption spectra of t-PA in the near-UV region. Difference spectra were obtained for sct-PA $(-)$ and tct-PA $(---)$, both at $0.09 \mathrm{mg} / \mathrm{ml}$, by subtracting spectra at $\mathrm{pH} 7.8$ from those at $\mathrm{pH} 11.5$ at $25^{\circ} \mathrm{C}$ in PBST in $1-\mathrm{cm}$ pathlength cells.
Fluorescence. The endogenous protein fluorescence spectra of sct-PA and tct-PA in PBST at $25^{\circ} \mathrm{C}$ are shown in Fig. 2. The spectrum for sct-PA is corrected for contamination with tct-PA; all subsequent fluorescence parameters are similarly corrected. Clearly, the intrinsic protein fluorescence of native tct-PA is less than that of native sct-PA. The quantum yields and emission maxima for sct-PA and tct-PA and their reduced, denatured forms are shown in Table I. The quantum yields measured by exciting at $280 \mathrm{~nm}$ are protein quantum yields and are produced by tryptophan and tyrosine fluorescence, whereas the quantum yields measured by exciting at $295 \mathrm{~nm}$ are produced primarily by tryptophan fluorescence. The tyrosine quantum yields were obtained by subtracting tryptophan from protein quantum yields and are also listed in Table I. The tryptophan quantum yields were derived using Eq. 4, in which the ratio of tryptophan to tyrosine was calculated from the known amino acid composition (29) and in which a value of 4 was used for the ratio $\epsilon_{\mathrm{trp}} / \epsilon_{\mathrm{tyr}}(30)$.

The protein quantum yield of native tct-PA is $54 \%$ of that of sct-PA, while the tryptophan quantum yield is $44 \%$ of that of the single chain form. With denaturation, protein quantum yields decrease for both sct-PA and tct-PA by equivalent amounts ( 27 and $21 \%$, respectively), whereas the tryptophan quantum yield decreases by $11 \%$ for sct-PA but does not change significantly for tct-PA. In the native form, the quantum yield of tyrosine is $40 \%$ greater in tct-PA than in sct-PA, whereas it is $52 \%$ greater in the corresponding denatured species.

The maximal emission wavelengths are comparable in all cases, approximating $360 \mathrm{~nm}$ for the native species. On denaturation, these emission maxima increase in each case, to the 364-369-nm range.

Fluorescence quenching by sodium iodide. The effect of increasing concentrations of the ionic fluorescence quencher iodide on the intrinsic fluorescence of t-PA is shown in Fig. 3, $A$ and $B$. The Stern-Volmer plots suggest that principally dynamic (collisional) processes contribute to quenching of intrinsic protein fluorescence. No upward curvature is noted, implying that the static quenching parameter is small. No significant downward curvature is noted, implying an absence of significantly different multiple emission components (31). The slope of the Stern-Volmer plots for native sct-PA and native tct-PA are similar, suggesting similar quencher access to fluorophores in these molecules.

Quenching is enhanced somewhat with reduction and alkylation, more with tct-PA than with sct-PA. Denaturation greatly enhances the quenching by iodide, particularly for the

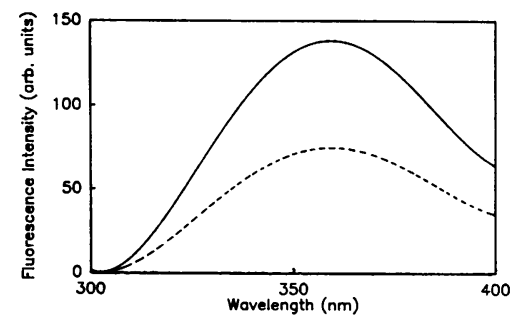

Figure 2. Protein fluorescence emission spectra of t-PA. Spectra were recorded for 0.009 $\mathrm{mg} / \mathrm{ml} \mathrm{sct}-\mathrm{PA}(-)$ and tct-PA $(---)$ in PBST at $25^{\circ} \mathrm{C}$. Samples were excited at $280 \mathrm{~nm}$ and emission was recorded at $360 \mathrm{~nm}$ with an excitation slit width of $1.25 \mathrm{~nm}$ and an emission slit width of $5.00 \mathrm{~nm}$. 
Table I. Protein, Tryptophan, and Tyrosine Quantum Yields of Recombinant Human $t-P A^{*}$

\begin{tabular}{cccc}
\hline & $Q_{\mathrm{p}}\left(\lambda_{\max }\right)^{\ddagger}$ & $Q_{\mathrm{up}}\left(\lambda_{\max }\right)^{\$}$ & $Q_{\mathrm{ryr}}$ \\
\hline Native $^{l l}$ & & & \\
sct-PA & $0.093(360)$ & $0.075(361)$ & 0.040 \\
tct-PA & $0.050(360)$ & $0.033(360)$ & 0.056 \\
Denatured" & & & \\
sct-PA & $0.068(364)$ & $0.067(365)$ & 0.032 \\
tct-PA & $0.039(367)$ & $0.035(369)$ & 0.021 \\
\hline
\end{tabular}

* The values of the quantum yields and emission maxima are averages obtained from two experiments performed in duplicate with deviations of $\pm 5 \%$ and $\pm 1 \mathrm{~nm}$, respectively. Values for sct-PA have been corrected for contaminant tct-PA as described in Methods.

‡ Excitation at $280 \mathrm{~nm}$.

${ }^{8}$ Excitation at $295 \mathrm{~nm}$.

"In PBST.

In $6 \mathrm{M}$ quanidine hydrochloride-PBST.

reduced, alkylated species. There appears to be no significant difference between sct-PA and tct-PA as to quenching by iodide in the denatured states.

Comparison of enzymatic activity of sct-PA and tct-PA. The hydrolytic activity of sct-PA and tct-PA against the chromogenic substrate $\mathrm{S}-2288$ was assessed in the presence and absence of SFM. As seen in Table II, sct-PA has a $K_{\mathrm{m}}$ for substrate that is 3.3-fold greater than tct-PA and a similar $k_{\text {cat }}$, yielding a catalytic efficiency $\left(k_{\text {cat }} / K_{\mathrm{m}}\right)$ that is only $26 \%$ of that of tctPA in the absence of SFM. In the presence of $33 \mu \mathrm{g} / \mathrm{ml}$ SFM, however, the $K_{\mathrm{m}}$ of both sct-PA and tct-PA decrease significantly, $\sim 16-$ and 4-fold, respectively. The $k_{\text {cat }}$ of both sct-PA and tct-PA decreased very slightly (16 and $19 \%$, respectively). These changes lead to a catalytic efficiency that is dramatically enhanced for sct-PA (13-fold) and significantly increased for tct-PA (3.5-fold). Most interestingly, with these changes in catalytic constants induced by SFM, sct-PA manifests a catalytic efficiency that is not significantly different from that of tct-PA: Importantly, predominantly sct-PA did not undergo any significant (auto)conversion to tct-PA during the course of these enzymatic measurements; this was demonstrated by inhibiting further enzymatic activity at the end of the reaction with 250 kallikrein inactivator units $/ \mathrm{ml}$ aprotinin, subjecting the reaction solution to gel exclusion chromatography on Sephadex G-100 in order to isolate t-PA from SFM and from chromogenic substrate, lyophilizing the t-PA peak, and conducting $\mathrm{NH}_{2}$-terminal amino acid analysis using a gas phase sequenator as described in Methods. By this kind of analysis, predominantly sct-PA (76\% single-chain at the outset of the reaction) remained as such throughout the reaction, both in the presence (75\%, mean of two measurements) and absence (76\%, mean of two measurements) of SFM. In addition, we used radioiodinated sct-PA in the reaction mixture and prepared the mixture with electrophoretic sample buffers with and without 2-ME immediately after adding aprotinin. Electrophoresis was performed on $10 \%$ gels and autoradiography was subsequently performed. Again, by densitometric analysis, there was no evidence for autoconversion of sct-PA to the two-chain form during the course of these very brief enzymatic reactions (75\% sct-PA, mean of two measurements in the absence of SFM; 75\% sct-PA, mean of two measurements in the presence of SFM).

Effect of poly-L-lysine and SFM on fluorescence intensity of eos-t-PA. To detect subtle changes in conformation that may accompany the binding of sct-PA to fibrin, we measured the effect of the binding of poly-L-lysine (PLL) and SFM on the steady state fluorescence intensity of eos-sct-PA and eos-tctPA. Because these protein species are labeled solely at a single sulfhydryl group in the region adjacent to the fibrin-binding domain (32), changes in the fluorescence of the reporter group probably reflect local changes in the microenvironment of the molecule important for fibrin binding (the finger domain and the kringle-2 segment).

As shown in Table III, eos-tct-PA has only $84 \%$ of the fluorescence intensity of eos-sct-PA at $540 \mathrm{~nm}$ when excited at $519 \mathrm{~nm}$. With addition of $250 \mu \mathrm{g} / \mathrm{ml}$ PLL or $75 \mu \mathrm{g} / \mathrm{ml} \mathrm{SFM}$ the fluorescence intensity of eos-sct-PA decreases by $\sim 13 \%$, approaching that of eos-tct-PA, whereas that of eos-tct-PA does not change significantly. Titrations of these fluorescence changes using PLL and SFM are shown in Fig. 4. Note that the data are expressed as the ratio of the change in fluorescence intensity at a given concentration of ligand to that at saturating concentrations of ligand to facilitate estimations of apparent $K_{\mathrm{d}}$. From these data, the apparent estimated $K_{\mathrm{d}}$ for PLL $\left(\left\langle M_{\mathrm{r}}\right\rangle\right.$ $=300,000)$ is $10 \mu \mathrm{g} / \mathrm{ml}$, whereas that for SFM is $11 \mu \mathrm{g} / \mathrm{ml}$.

Binding of $t$-PA to FM-Matrex. We used FM-Matrex as a ligand for t-PA binding experiments to facilitate estimations of affinity and stoichiometry. Because fibrin monomer on Matrex beads cannot polymerize, the nature of the ligand is known and stoichiometry can be determined. In addition, with fibrin monomer covalently attached to beads, bound t-PA can be readily separated from free t-PA by centrifugation. The binding data obtained with this system are shown in Fig. 5 . Clearly, both sct-PA and tct-PA bind saturably and specifi-

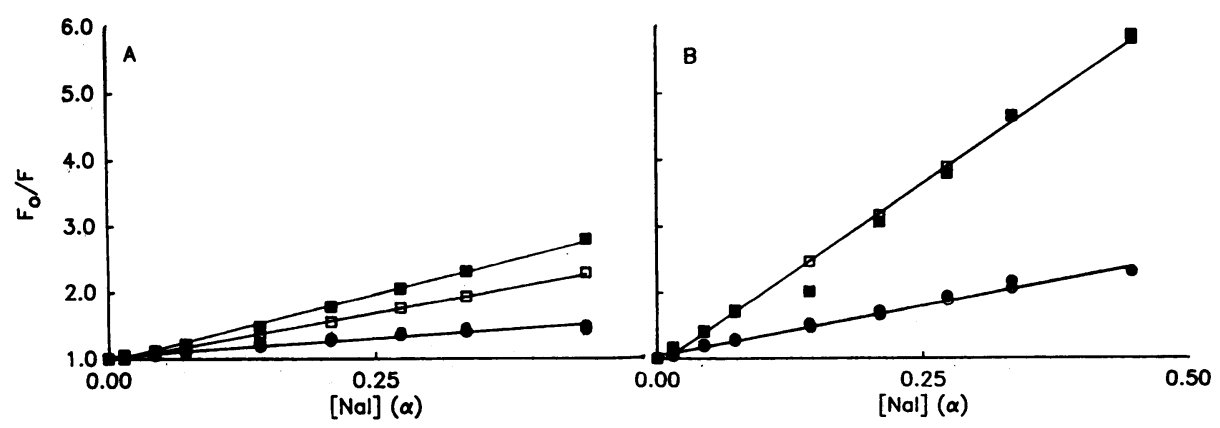

Figure 3. Quenching of protein fluorescence of t-PA by iodide. Stern-Volmer plots were constructed for sct-PA (open symbols) and tct-PA (closed symbols) in native (circles) and reduced, alkylated (squares) species in the absence $(A)$ and presence $(B)$ of $6 \mathrm{M}$ guanidine hydrochloride. Quenching by iodide $(A$ and $B$ ) was measured in PBST at $25^{\circ} \mathrm{C}$ on excitation with $280 \mathrm{~nm}$ and emission at $360 \mathrm{~nm}$. 
Table II. Kinetic Constants for Hydrolysis of S-2288 by sct-PA and tct-PA*

\begin{tabular}{lllr}
\hline & \multicolumn{1}{c}{$K_{\mathrm{m}}$} & $k_{\text {cat }}$ & \multicolumn{1}{c}{$k_{\text {cal }} / K_{\mathrm{m}}$} \\
\hline & $m M$ & $s^{-1}$ & $\mathrm{mM}^{-1} / \mathrm{s}^{-1}$ \\
& & & \\
Sct-PA $^{\ddagger}$ & & & \\
$\quad$ Without SFM & 0.82 & 6.75 & 8.23 \\
$\quad$ With SFM & 0.051 & 5.53 & 108.43 \\
tct-PA & & & \\
Without SFM & 0.25 & 7.82 & 31.28 \\
With SFM & 0.061 & 6.57 & 107.71 \\
\hline
\end{tabular}

* Enzymatic assays and analyses of data were performed as described in Methods.

${ }^{\ddagger}$ Kinetic constants for sct-PA have been corrected for contaminant tct-PA as described in Methods.

cally, and both bind similarly with an approximate apparent $K_{\mathrm{d}}$ of $3.9 \mu \mathrm{M}$. With $4 \times 10^{5}$ molecules of fibrinogen bound per bead (see Methods), at saturation, the stoichiometry of t-PA to fibrin monomer is $\sim 1.1: 1$.

\section{Discussion}

In most published studies, t-PA activity is measured indirectly as plasmin activity $(9,33)$, and because plasmin itself can convert sct-PA to tct-PA, any quantitative assessment of t-PA activity is confounded by ongoing conversion of enzyme by substrate. Furthermore, absolutely pure preparations of sct-PA are not available and there is, as yet, no inhibitor that selectively inhibits contaminant tct-PA. We have attempted to overcome these difficulties in the experiments described here by several significant experimental alterations. We used the chromogenic substrate S-2288 to measure the kinetic constants of t-PA. In addition, however, we have derived the actual constants of sct-PA using preparations containing tct-PA by quantitative assessment of the degree of contamination. We also used a different preparation of fibrin for assessing activation. Instead of Desafib (American Diagnostica), which may manifest altered conformations and thereby have altered acti-
Table III. Fluorescence Emission Intensity of Eos-t-PA*

\begin{tabular}{cc}
\hline & Normalized emission intensity \\
\hline & $\%$ \\
Eos-sct-PA $^{\ddagger}$ & 100 \\
+ Poly-L-lysine & 87 \\
+ SFM" & 85 \\
Eos-tct-PA $^{\ddagger}$ & 84 \\
+ Poly-L-lysine & 83 \\
+ SFM & 83 \\
\hline
\end{tabular}

* Measurements were made in PBST at $25^{\circ} \mathrm{C}$ with excitation at 519 $\mathrm{nm}$ and emission at $540 \mathrm{~nm}$. Each value represents the average of two experiments.

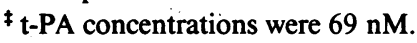

${ }^{8}$ Poly-L-lysine was added to a concentration of $250 \mu \mathrm{g} / \mathrm{ml}$.

"SFM was added to a concentration of $75 \mu \mathrm{g} / \mathrm{ml}$.

vating ability, we used SFM maintained in the monomeric state with the tetrapeptide inhibitor GPRP. When we attempted the kinetic experiments with Desafib, we observed only marginal activation compared with SFM.

We complemented these kinetic studies with structural studies of both sct-PA and tct-PA. Using both UV absorption and fluorescence spectroscopy of these molecules, we showed that there were both similarities and dramatic differences between single- and two-chain species in both the native and denatured states. What specific features of the protein conformation are affected by conversion of sct-PA to tct-PA cannot be determined from these data because the interpretations are limited by the fact that the measured signals represent averages from fluorophores located in multiple regions of a given protein molecule.

Despite these shortcomings, we were able to derive some useful general information about the similarities and differences between the conformations of sct-PA and tct-PA. The UV difference absorption spectra were very nearly identical. Since the absorption differences induced by alkaline denaturation are similar, these data argue that the microenvironments of the subclass of intrinsic chromophores perturbed by alkalinity must be similar.

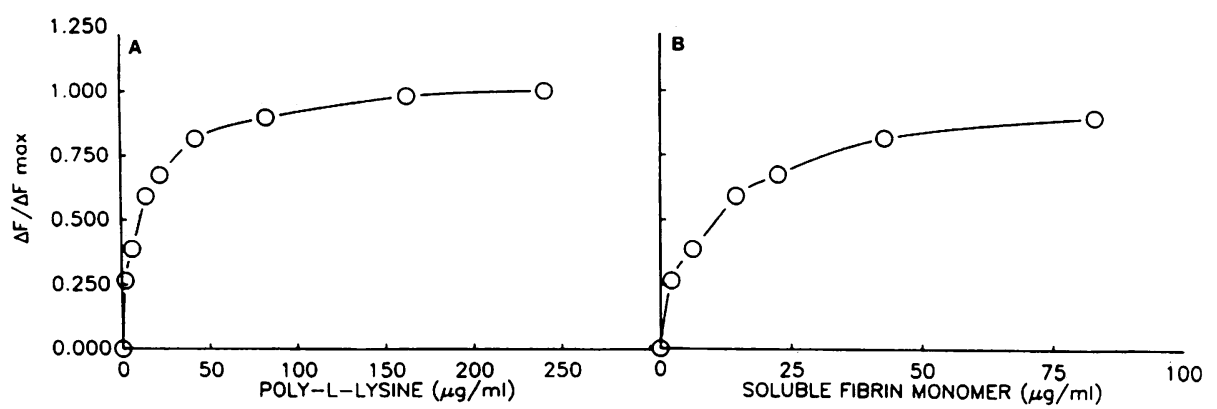

Figure 4. Effect of addition of poly-Llysine and SFM on eos-t-PA fluorescerice. Fluorescence was measured for solutions of sct-PA at $0.005 \mathrm{mg} / \mathrm{ml}$ in PBST at $25^{\circ} \mathrm{C}$. Samples were excited at $519 \mathrm{~nm}$ and emission was recorded at $540 \mathrm{~nm}$ with an excitation slit width of $1.25 \mathrm{~nm}$ and an emission slit width of $5.00 \mathrm{~nm}$. The ordinate is defined by the ratio of the change in fluorescence intensity at any given concentration of poly-L-lysine or SFM to the maximal change in fluorescence intensity. At saturating concentrations of ligand, the absolute fluorescence intensity is reduced relative to that in the absence of ligand. The data are plotted in this fashion to facilitate approximate binding analyses. 


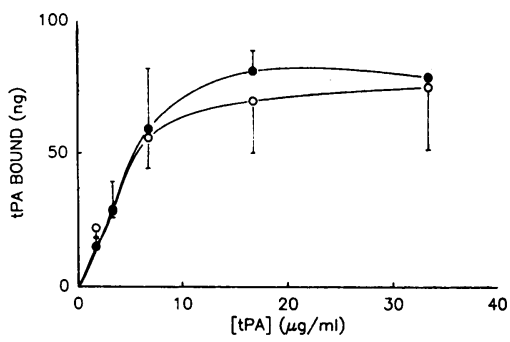
(1) performed for 20 min with rocking. Beads were collected by centrifugation at $8,700 \mathrm{~g}$ for $2 \mathrm{~min}$ at the end of the incubation period, washed twice with PBST, and bound radioactivity was determined. Specific binding (plotted here) was distinguished from nonspecific binding by addition of a 20 -fold excess of unlabeled t-PA to the incubation medium. Nonspecific binding amounted to no more than $35 \%$ of total bound counts.

In contrast, analysis of intrinsic protein fluorescence reveals differences between sct-PA and tct-PA. The fluorescence of t-PA predominantly originates from tryptophan moieties. Despite the presence of 24 tyrosine residues per 72,000-D molecule and only 13 tryptophan residues, the tryptophan fluorescence dominates protein fluorescence. Dramatic reductions in the quantum yield of intrinsic protein fluorescence accompany the conversion of sct-PA to tct-PA. In addition, the polar quencher iodide had greater access to fluorophores in reduced, alkylated, nondenatured two-chain than in single-chain forms.

The wavelengths of the emission maxima of sct-PA and tct-PA are also rather similar and suggest that most of the tryptophan residues are relatively exposed to solvent rather than buried in the hydrophobic matrix of the protein. Denaturation tends to shift the maxima to slightly longer wavelengths and to decrease the quantum yield. Although neither the emission maximum nor the quantum yield can be a simple function of the degree of exposure of tryptophan residues (21, 34 ), the combination of a modest red shift and a decrease in quantum yield argues that some tryptophan residues buried in the native molecule are exposed after denaturation.

Although both sct-PA and tct-PA bound to fibrin monomer covalently linked to Matrex beads identically, significant differences in the vicinity of the fibrin-binding domain can be inferred from the differences in fluorescence emission of eosin covalently linked to Cys 83 . The fluorescence emission of this fluorophore was reduced by $16 \%$ in tct-PA compared with sct-PA. With addition of PLL or SFM to a solution of sct-PA the fluorescence of the eosin label decreased, approaching that in tct-PA. Addition of PLL or SFM had no effect on eosin fluorescence in tct-PA.

Together, these structural and kinetic data suggest that sctPA undergoes a conformational change on binding in a oneto-one complex to fibrin monomer. This conformational change in turn leads to dramatic differences in catalytic efficiency of the single-chain species, primarily by a significant reduction in $K_{\mathrm{m}}$. In so doing, fibrin-bound sctPA assumes the kinetic profile of tct-PA bound to fibrin and, at least in the fibrin-binding domain as reported by eosin bound to Cys 83 , adopts a conformation approaching that of tct-PA. These data argue that sct-PA is more fibrin-specific than tct-PA primarily because of its markedly reduced catalytic efficiency in the ab- sence of fibrin. Despite similarities in fibrin affinity, fibrin binding induces a marked enhancement in the catalytic efficiency of sct-PA. Fibrin binding also increases the catalytic efficiency of tct-PA, but the extent of enhancement is much greater for the single chain form (3.5-fold vs. 13-fold). These conformation-dependent enzymatic differences may account for the observed differences between predominantly sct-PA and predominantly tct-PA preparations observed in vivo (35).

\section{Acknowledgments}

The author wishes to thank Irina Barsky-Vasserman, Deborah Smick, and Patricia Amarante for excellent technical assistance; Drs. Douglas E. Vaughan, Michael E. Mendelsohn, Allen J. Naftilan, and M. Audrey Rudd for helpful comments and discussion; and Dr. Kenneth Bridges for assistance in the densitometric measurements.

This work was conducted during Dr. Loscalzo's tenure as a Clinician-Scientist of the American Heart Association. This work was supported in part by National Institutes of Health grant HL-40411 and by a Grant-in-Aid from the American Heart Association, Massachusetts affiliate.

\section{References}

1. Verstraete, M., and D. Collen. Thrombolytic therapy in the eighties. Blood. 67:1529-1541.

2. Vehar, G. A., W. J. Kohr, W. F. Bennett, D. C. Pennica, A Ward, R. N. Harkins, and D. Collen. 1984. Tissue plasminogen activator. Biotechnology. 2:1051-1056.

3. Andreasen, P. A., L. S. Nielsen, J. Grondahl-Hansen, L. Skriver, J. Zeuthen, R. W. Stephes, and K. Dano. 1984. Inactive proenzyme to tissue-type plasminogen activator from human melanoma cells, identified after affinity purification with a monoclonal antibody. EMBO (Eur. Mol. Biol. Organ.) J. 3:51-56.

4. Ichinose, A., W. Kisiel, and K. Fujikawa. 1984. Proteolytic activation of tissue plasminogen activator by plasma and tissue enzymes. FEBS (Fed. Eur. Biochem. Soc.) Lett. 175:412-418.

5. Tate, K. M., D. L. Higgins, W. E. Holmes, M. E. Winkler, H. L. Heyneker, and G. A. Vehar. 1987. Functional role of proteolytic cleavage at arginine-275 of human tissue plasminogen activator as assessed by site-directed mutagenesis. Biochemistry. 26:338-343.

6. Rijken, D. C., and D. Collen. 1981. Purification and characterization of the plasminogen activator secreted by human melanoma cells in culture. J. Biol. Chem. 256:7035-7041.

7. Wallen, P., M. Ranby, N. Bergsdorf, and P. Kok. 1981. Purification and characterization of tissue plasminogen activator: on the occurrence of two different forms and their enzymatic properties. Prog. Fibrinolysis. 5:16-23.

8. Rijken, D. C., M. Hoylaerts, and D. Collen. 1982. Fibrinolytic properties of one-chain and two-chain human extrinsic (tissue-type) plasminogen activator. J. Biol. Chem. 257:2920-2925.

9. Ranby, M. 1982. Studies on the kinetics of plasminogen activation by tissue plasminogen activator. Biochim. Biophys. Acta. 704:461-469.

10. Ranby, M., N. Bergsdorf, G. Pohl, and P. Wallen. 1982. Isolation of two variants of native one-chain tissue plasminogen activator. FEBS (Fed. Eur. Biochem. Soc.) Lett. 146:289-292.

11. Ranby, M., N. Bergsdorf, B. Norrman, E. Suenson, and P. Wallen. 1982. Tissue plasminogen activator kinetics. Prog. Fibrinolysis. 6:182-184.

12. Laudano, A. P., and R. F. Doolittle. 1980 . Studies on synthetic peptides that bind to fibrinogen and prevent fibrin polymerization: structural requirements, number of binding sites, and species differences. Biochemistry. 19:1013-1019.

13. Loscalzo, J., A. Inbal, and R. I. Handin. 1986. Von Willebrand protein facilitates platelet incorporation in polymerizing fibrin. J. Clin. Invest. 78:1112-1119.

14. Banyai, L., A. Varadi, and L. Patthy. 1983. Common evolu- 
tionary origin of the fibrin-binding structures of fibronectin and tissue-type plasminogen activator. FEBS (Fed. Eur. Biochem. Soc.) Lett. 163:37-41.

15. Franzen, J. S., P. S. Marchetti, and D. S. Feingold. 1980. Resonance energy transfer between catalytic sites of bovine liver uridine dephosphoglucose dehydrogenase. Biochemistry. 19:6080-6089.

16. Coller, B. S. 1980. Interaction of normal, thrombasthenic, and Bernard-Soulier platelets with immobilized fibrinogen-defective platelet-fibrinogen interaction in thrombasthenia. Blood. 55:169-178.

17. Bennett, J. S., and G. Vilaire. 1979. Exposure of platelet fibrinogen receptors by ADP and epinephrine. J. Clin. Invest. 64:13931401.

18. Loscalzo, J., and D. E. Vaughan. 1987. Human tissue-type plasminogen activator facilitates platelet disaggregation. J. Clin. Invest. 79:1749-1755.

19. Parker, C. A., and W. J. Rees. 1960. Correction of fluorescence spectra and measurement of fluorescence quantum efficiency. Analyst (Lond.). 85:587-600.

20. Teale, F. W. J., and G. Weber. 1957. Ultraviolet fluorescence of the aromatic amino acids. Biochem. J. 65:476-482.

21. Kronman, M. J., and L. G. Holmes. 1971. The fluorescence of native, denatured, and reduced-denatured proteins. Photochem. Photobiol. 14:113-134.

22. Ellman, G. L. 1959. Tissue sulfhydryl groups. Arch. Biochem. Biophys. 82:70-72.

23. Habeeb, A. F. S. A. 1966. Chemical evaluation of conformational differences in native and chemically modified proteins. Biochim. Biophys. Acta. 115:440-454.

24. Collier, H. B. 1973. A sensitive method for localization of disulfide containing peptides in column effluents. Anal. Biochem. 56:310-311.

25. Weber, K., and M. Osborne. 1969. The reliability of molecular weight determinations by dodecyl sulfate-polyacrylamide gel electrophoresis. J. Biol. Chem. 244:4406-4412.

26. Laemmli, U. K. 1970. Cleavage of structural proteins during the assembly of the head of bacteriophage T4. Nature (Lond.). 227:680-682.

27. Lowry, O. H., N. J. Rosebrough, A. L. Farr, and R. Randall. 1951. Protein measurement with the Folin reagent. J. Biol. Chem. 193:265-275.

28. Jaffe, H. H., and M. Orchin. 1962. Theory and Application of Ultraviolet Spectroscopy. John Wiley \& Sons, Inc., New York. 592.

29. Pennica, D., W. E. Holmes, W. J. Kohr, R. N. Harkins, G. A. Vehar, C. A. Ward, W. F. Bennett, E. Yelverton, P. H. Seeburg, H. L. Heyneker, D. V. Goeddel, and D. Collen. 1983. Cloning and expression of human tissue-type plasminogen activator cDNA in E. coli. Nature (Lond.). 301:214-217.

30. Weber, G., and L. B. Young. 1964. Fragmentation of bovine serum albumin by pepsin. J. Biol. Chem. 239:1424-1431.

31. Lehrer, S. S., and P. C. Leavis. 1978. Solute quenching of protein fluorescence. Methods Enzymol. 49:222-236.

32. Ichinose, A., K. Takio, and K. Fujikawa. 1986. Localization of the binding site of tissue-type plasminogen activator to fibrin. J. Clin. Invest. 78:163-169.

33. Christensen, U., and S. Mullertz. 1977. Kinetic studies on the urokinase catalyzed conversion of $\mathrm{NH}_{2}$ terminal lys-plasminogen to plasmin. Biochim. Biophys. Acta. 480:275-281.

34. Weinryb, I., and R. F. Steiner. 1970. Luminescence of tryptophan and tyrosine residues of papain in solution. Biochemistry. 9:135-146.

35. Garabedian, H. D., H. K. Gold, R. C. Leinbach, J. C. Johns, T. Yasuda, M. Kanke, and D. Collen. 1987. Comparative properties of two clinical preparations of recombinant human tissue-type plasminogen activator in patients with acute myocardial infarction. J. Am. Coll. Cardiol. 9:599-607. 